\title{
Influence of sensitivity on response bias in taste and audition
}

\author{
JENNIFER A. STILLMAN \\ Massey University Albany, Auckland, New Zealand \\ GEOFFREY M. BROWN \\ University of Auckland, Auckland, New Zealand \\ and \\ TOM TROSCIANKO \\ University of Bristol, Bristol, England
}

\begin{abstract}
A detection theoretic analysis was employed to examine sensitivity and response bias in two modalities. In Experiment 1, 6 tasters made same-different judgments about the concentration of either sucrose or quinine in pairs of tonic water samples. The beverages were colored, but color was not predictive of the concentration of the sweet or bitter ingredient. When same-different ratings were collapsed to approximate the outcome of a categorical decision, tasters with poorer sensitivity appear to have adopted more extreme response criteria than did tasters with greater sensitivity, irrespective of taste quality, color, or whether pairs of solutions comprised the same or different colors. In Experiment 2 , 3 individuals discriminated pairs of $1000-\mathrm{Hz}$ sinusoids differing in amplitude. Six amplitude differences were tested. Rating-scale versions of two paradigms: The single-interval yes-no task and the twointerval same-different task were used to measure sensitivity and bias. There was a preponderance of "same" responses in the same-different task. Estimates of bias obtained from collapsed ratings in both tasks were unaffected by sensitivity, but a consideration of the range over which sets of criteria were spread suggested a general tendency toward more conservative response biases as sensitivity declined.
\end{abstract}

The outstanding contribution of detection theory to psychophysical measurement is its ability to provide measures of sensitivity that are independent across manipulations of response bias, and vice versa. Thus, a number of recent papers evaluating both parametric and "nonparametric" indices of response bias agree on theneed to select an index displaying this property (Donaldson, 1992; Macmillan \& Creelman, 1990, 1996; See, Warm, Dember, \& Howe, 1997; Snodgrass \& Corwin, 1988). On occasion, however, researchers overlook the fact that statistical independence does not imply behavioral independence. For example, Snodgrass and Corwin (1988), who were scrupulous in their choice of bias measures, concluded that different dementias produced characteristic response biases. The recognition memory task in which bias was measured in their study was markedly more difficult for some groups than for others; thus, the conclusion presupposes the behavioral independence of bias and sensitivity. In a set of three experiments, See et al.

The authors thank Michael Hautus for making available the computer program from which the $d^{\prime}$ estimates were obtained and Douglas Creelman, Sandy MacRae, and one anonymous reviewer for their valuable suggestions on an earlier version of this manuscript. Correspondence should be addressed to J. A. Stillman, School of Psychology, Massey University Albany, Private Bag 102-904, North Shore MSC, Auckland, New Zealand (e-mail: j.a.stillman@massey.ac.nz).
(1997) examined five detection theory measures of response bias using a vigilance task involving the detection of small increments in the height of a single white line flashed on a computer screen. In their Experiment 2, as in Snodgrass and Corwin's experiment, three payoffs (conservative, neutral, and liberal) were each enforced under two levels of difficulty, referred to as low and high salience. See et al. commented, "The presence of either a main effect or an interaction involving salience would signify an undesirable association between bias and signal salience, which theoretically should not covary" (p. 20).

In an unpublished study concerned with the influence of color on flavor perception and on response bias, we (Stillman \& Troscianko, 1996) found that, despite the independence of measures of sensitivity and bias, it was likely that those characteristics were not independent with respect to behavior. In that study, we were unable to establish whether color directly affected taste perception; however, we report, as Experiment 1 below, that aspect of the study concerned with response bias. In Experiment 2, listeners discriminated pairs of $1000-\mathrm{Hz}$ sinusoids across six amplitude differences, to explore further the nature of the relationship between sensitivity and response bias, this time in the absence of any externatl biasing influences. Sensitivity and response bias were explored using the following rating tasks: the two-interval same-different task 
(in Experiments 1 and 2) and the single-interval yes-no task (in Experiment 2). Response bias was specified using the index $c$, or an analogous measure $c_{\mathrm{sd}}$. The index $c$ has been the most strongly supported bias measure in recent investigations (Macmillan \& Creelman, 1990; See et al., 1997); however, we found no studies in which its same-different counterpart has been specifically evaluated against other candidate measures.

The nature of the relationship between sensitivity and response bias was examined in order to shed light on the validity of response bias comparisons when sensitivity is not equivalent across the individuals or groups being compared. Difficulties with the concurrent measurement of sensitivity and response bias are discussed.

\section{EXPERIMENT 1}

Color is known to be an important aspect in the perception of foods and beverages, influencing identification, acceptability, and reported taste quality (e.g., DuBose, Cardello, \& Maller, 1980; Norton \& Johnson, 1987; Stillman, 1993; Tuorila-Ollikainen, 1982). In order to examine the influence of color, we introduced irrelevant color while measuring sensitivity to variations in the concentration of sucrose and quinine in tonic water. The colors used-green and yellow for beverages in which the concentration of the sweet-tasting ingredient (sucrose) was varied, and green and red for beverages in which the concentration of the bitter-tasting ingredient (quinine) was varied - were chosen because they appeared, on the basis of previously published studies, to influence judgments of taste quality. Green-colored beverages are generally associated with decreased sweetness perception compared with yellow-colored beverages (Pangborn \& Hansen, 1963; Roth, Radle, Gifford, \& Clydesdale, 1988 ), and red solutions have been associated with reduced bitterness relative to green solutions (Maga, 1974). Aside from the influence of specific colors, we expected that, when same-different taste judgments were called for, visual similarity would influence responding. To anticipate the conclusion, we found that visual similarity and specific color were both less influential in determining response bias than was sensitivity.

\section{Method}

\section{Participants}

Six volunteers, 2 men and 4 women, students at the University of Bristol, were paid for their participation. Their average age was 25 years. All six had normal color vision as assessed by Ishihara's (1964) Tests for Color Blindness (38-plates edition). None of the participants had previous experience in taste experiments.

\section{Stimuli}

Gustatory stimuli. The solutions to be discriminated comprised samples of a degassed commercially available tonic water. The beverage was initially uncolored and had a complex flavor incorporating dimensions of both sweetness and bitterness. The product was sweetened with sucrose alone, without the addition of artificial sweeteners. Two sets of solutions were prepared. In one set, there were two concentrations of sucrose, each of which could be colored either yellow or green. In the other set, there were two concentrations of quinine, each of which could be colored either red or green. Color was not predictive of the concentration of the sweet or bitter ingredient.

Tonic water from the same batch, stored in the dark, was used throughout. Solutions were prepared at least $14 \mathrm{~h}$ in advance and were served at room temperature, held constant at approximately $21.5^{\circ} \mathrm{C}$. The original concentrations of sucrose and quinine in the tonic water were as follows: sucrose, $5.4 \%$ weight/volume; quinine $0.008 \%$ weight/volume. When sucrose was varied, half of the samples were made up with the addition of $28 \mathrm{~g}$ per liter of food-grade sucrose. The volume of liquid in the no-added-sugar and addedsugar versions was equalized prior to the addition of food colorings. When quinine was varied, half of the samples were made up with the addition of $30 \mathrm{ml}$ of an aqueous solution of pharmaceuticalgrade quinine monosulphate dihydrate, $1 \mathrm{mg}$ per milliliter. The volume of the beverage from which the remaining samples were obtained was equalized by the addition of $30 \mathrm{ml}$ of water. All water used in the study was filtered so as to remove organic chemicals, chlorine, and heavy metals.

Visual stimuli. Commercial food colorings (tartrazine, Green S, Sunset Yellow, Ponceau 4R, Carmoisene, Quinoline Yellow) were blended to produce predetermined hues as specified below. The colorings were made up to $5 \mathrm{ml}$ with water before being added to each liter of tonic.

The perceptual attributes of the samples were evaluated using data from Troscianko $(1979,1982)$. Measurements of the luminance and chromaticity of the samples were made using a Spot Chroma Meter (Minolta model CS-100). Colorimetric values of the samples were as follows: red, $u^{\prime}=0.369, v^{\prime}=0.502, Y / Y_{0}=0.329$; green, $u^{\prime}=0.153, v^{\prime}=0.532, Y / Y_{0}=0.629 ;$ and yellow, $u^{\prime}=0.237, v^{\prime}=$ $0.555, Y / Y_{0}=0.932$.

On every trial, two $10-\mathrm{ml}$ samples of the colored solutions were presented in neutral, translucent, $30-\mathrm{ml}$ polythene beakers placed side by side on a white tray located about $45 \mathrm{~cm}$ from the participant's eyes, directly below a pair of daylight fluorescent tubes.

\section{Procedure}

In view of the complex nature of tonic water, the precise sensory outcome of variations in sugar or quinine is unpredictable, although mixture suppression is commonly observed (Walters, 1996). Because the sensory changes produced by different combinations of sugar and quinine might be reported variously as either a decrease in bitterness or an increase in sweetness, we measured differential sensitivity using a same-different task that did not require a judgment about the sweetness or bitterness of the samples (Stillman \& Irwin, 1995). A rating method was used in conjunction with this task, in order to generate data from which reliable estimates of the detection theoretic index of sensitivity, $d^{\prime}$, could be calculated.

Each participant was tested individually and attended a total of 16 sessions lasting up to $1 \mathrm{~h}$ each. Testing with sucrose was completed before testing with quinine began. Excluding practice, each participant judged a total of 224 pairs of beverages in each phase of the experiment. An experimental session comprised two blocks of 16 trials separated by a rest period. Unique random permutations of all 16 possible orderings of the stimuli were generated by computer for every half-session. Thus, in each phase of the experiment, every combination of concentration and color was sampled twice during a block of trials. The first sessions with both sucrose and quinine were dedicated to practice. The solutions were always sampled from left to right and were expectorated, with between-trial, but not withintrial, rinses of filtered water being taken.

The participants were required to rate their confidence that the samples were the same with respect to sucrose or quinine concentration, as appropriate, using a numerical scale printed on a card in 
front of them, together with verbal definitions as follows: 1, certain different: 2 , fairly certain different; 3 , unsure, probably different; 4 , unsure, probably same; 5 , fairly certain same; 6 , certain same. After making their judgment orally, they were given feedback as to whether the samples were of the same or different sucrose (Phase 1) or quinine (Phase 2) concentrations. The participants were informed that we were interested in sensitivity to sweetness and bitterness but also in the effect of color as a context. They were also told that color had no predictive value with respect to the judgments they were required to make and that the a priori probability of same and different pairs was $p=.5$.

\section{Results}

\section{Receiver Operating Characteristics}

The ratings provided by the participants were pooled and used to construct receiver operating characteristics (ROCs) from which $d^{\prime}$ was derived. To this end, the hit rate was defined as the probability of a participant's choosing a particular rating (or a larger one) given that the two samples were of the same sucrose (or quinine) concentration, and the false-alarm rate was defined as the probability of a taster choosing the same rating given that the two samples were of different sucrose (or quinine) concentrations. The differencing model (Macmillan \& Creelman, 1991; Noreen, 1981) was adopted on the basis of previous research in taste (Hautus \& Irwin, 1995; Irwin, Stillman, Hautus, \& Huddleston, 1993; Stillman \& Irwin, 1995). This model assumes that the response on each trial is based on the difference between the sensory effects produced by each of the samples that are presented for comparison. ROCs that are asymmetrical about the negative diagonal of the ROC square are expected from this model.

The detection-theory index of discriminability, $d^{\prime}$, was estimated by a maximum likelihood procedure (Dorfman \& Alf, 1969) using the pooled ratings from each phase of the experiment. In order to examine the influence of color, we had intended to combine the responses of all six par- ticipants; however, 3 of the participants were clearly less sensitive to variations in the concentration of both the sweet and the bitter ingredient than were the remaining 3 participants. When sucrose was used, the average of individual $d$ ' estimates from the 3 "more sensitive" participants was $d^{\prime}=2.4$, and that from the 3 "less sensitive" participants was $d^{\prime}=0.93$. When quinine was used, the comparable averages were $d^{\prime} \mathrm{s}=1.75$ and 0.56 , respectively. For the subsequent analyses, sets of $d^{\prime}$ estimates were obtained from the pooled data of the less and more sensitive participants separately, as well as from the pooled data of all participants. Although all combinations of the variables were used simultaneously within a trial block, four separate analyses were undertaken with the data sorted according to color and according to whether the members of the pair presented for judgment were the same or different colors. In addition to the procedure for obtaining $d$ ' estimates outlined above, the "jackknife" method recommended by Irwin, Hautus, and Stillman (1992) for fitting ROCs to pooled ratings was also applied to the data. Three of the 26 sets of ratings could not be jackknifed because 1 or more of the participants did not allocate any responding to one of the two extreme categories. For the 23 sets of ratings that were jackknifed, the estimates from the two methods were equivalent (average difference between the pooled and jackknifed $d^{\prime}$ estimates $=0.02, S D=0.04$ ). For consistency, therefore, the pooled estimates were used in all subsequent analyses.

Table 1 summarizes the sensitivity estimates from each phase of the experiment. When the participants discriminated differences in sucrose concentration, the differencing model is a satisfactory fit, at the .05 level, to each set of data. Within each group of participants, values of $d^{\prime}$ are similar across conditions, despite differences in the number of trials contributing to particular estimates. The agreement of the various $d^{\prime}$ estimates from the com-

Table 1

Best-Fitting Values of $d^{\prime}$ for Judgments of Either Sucrose or Quinine Concentration, Along With $\chi^{2}$ Goodness-of-Fit Statistics

\begin{tabular}{|c|c|c|c|c|c|}
\hline \multirow[b]{2}{*}{ Stimuli } & \multirow{2}{*}{$\begin{array}{l}\text { Number } \\
\text { of Trials }\end{array}$} & \multicolumn{2}{|c|}{ Sucrose } & \multicolumn{2}{|c|}{ Quinine } \\
\hline & & $d^{\prime}$ & $\chi^{2}$ & $d^{\prime}$ & $\chi^{2}$ \\
\hline \multicolumn{6}{|c|}{ All Participants } \\
\hline All pairs & 1,344 & 1.77 & 7.89 & 1.19 & $10.35^{*}$ \\
\hline Same-colored pairs & 672 & 1.77 & 6.10 & 1.20 & $10.23^{*}$ \\
\hline Different-colored pairs & 672 & 1.77 & 2.94 & 1.04 & 4.39 \\
\hline Yellow (sucrose) or Red (quinine) pairs & 336 & 1.80 & 7.17 & 1.43 & 4.79 \\
\hline Green pairs & 336 & 1.74 & 2.00 & 0.96 & 6.26 \\
\hline \multicolumn{6}{|c|}{ More Sensitive Participants } \\
\hline Same-colored pairs & 336 & 2.40 & 9.09 & 1.50 & $19.56 * *$ \\
\hline Different-colored pairs & 336 & 2.47 & 2.85 & 1.38 & 7.38 \\
\hline Yellow (sucrose) or Red (quinine) pairs & 168 & 2.47 & 8.26 & 1.67 & 4.39 \\
\hline Green pairs & 168 & 2.32 & 3.26 & 1.36 & $15.16^{* *}$ \\
\hline \multicolumn{6}{|c|}{ Less Sensitive Participants } \\
\hline Same-colored pairs & 336 & 0.93 & 6.79 & 0.68 & 2.26 \\
\hline Different-colored pairs & 336 & 0.79 & 2.34 & 0.51 & 1.83 \\
\hline Yellow (sucrose) or Red (quinine) pairs & 168 & 0.82 & 4.26 & 1.05 & 4.20 \\
\hline Green pairs & 168 & 1.07 & 5.21 & 0.00 & 3.55 \\
\hline
\end{tabular}


bined data, despite variations in numbers of trials from 1,344 down to 336 , suggests there is sufficient reliability in the data when they are divided into two smaller sets. When the participants discriminated differences in quinine concentration, the $\chi^{2}$ values for the combined data reflect the fact that the model describes the data from the more sensitive participants less well. Even so, within-group estimates of sensitivity are comparable across conditions, so that, in the two cases where the model describes the data from the sensitive participants poorly, the estimates are likely to be an adequate reflection of sensitivity. Withingroup variability in $d^{\prime}$ appears greater with quinine than with sucrose.

\section{Response Bias}

Under the differencing strategy, the two observations on a trial are subtracted by the participant and compared with a difference criterion, with reference to which a judgment of "same" or "different" is made. Thus, three underlying normal distributions are implied, each with variance two (Macmillan \& Creelman, 1991). When both samples are of the same concentration, the distribution of differences will have a mean of zero. We will refer to this as the center distribution. When the two samples are of different concentrations and the weaker concentration is sampled first, the distribution of differences will have a mean of $-d^{\prime}$. When the stronger concentration is sampled first, the distribution will have a mean of $d^{\prime}$. Each rating category, therefore, is associated with a pair of criteria flanking the mean of the "same" distribution. However, under the assumption that the participant is concerned with the size and not the direction of difference, the criterion can be specified as a distance from the intersection of the "same" distribution with either "different" distribution. Following Macmillan and Creelman (1991, p. 153), this criterion is analogous to the $c$ of the yes-no paradigm and is referred to as $c_{\mathrm{sd}}$. Positive values of $c_{\mathrm{sd}}$ reflect a bias toward "same" responses. A maximum bias toward "different" responses occurs when the criteria meet at the mean of the "same" distribution.

When investigating bias in a rating experiment, one approach is to treat the participant's ratings as if they were obtained using only two response alternatives. On the 6point scale used in this investigation ratings of 1,2 , and 3 comprise one alternative ("different"), and ratings of 4 , 5 , and 6 comprise the other ("same"). The data then reduce to a single hit and false-alarm pair analogous the outcome of a categorical judgment. This datum provides an indication of the point at which the participant is equally disposed to opt for either alternative. A disadvantage of this approach is that the measure may equate the biases of individuals, or groups, whose category boundaries range over vastly different distances. To provide more information about response bias, the two extreme response categories were used to define the response range.

The two panels of Figure 1 graphically represent the outcomes of Experiment 1. The symbols have the same
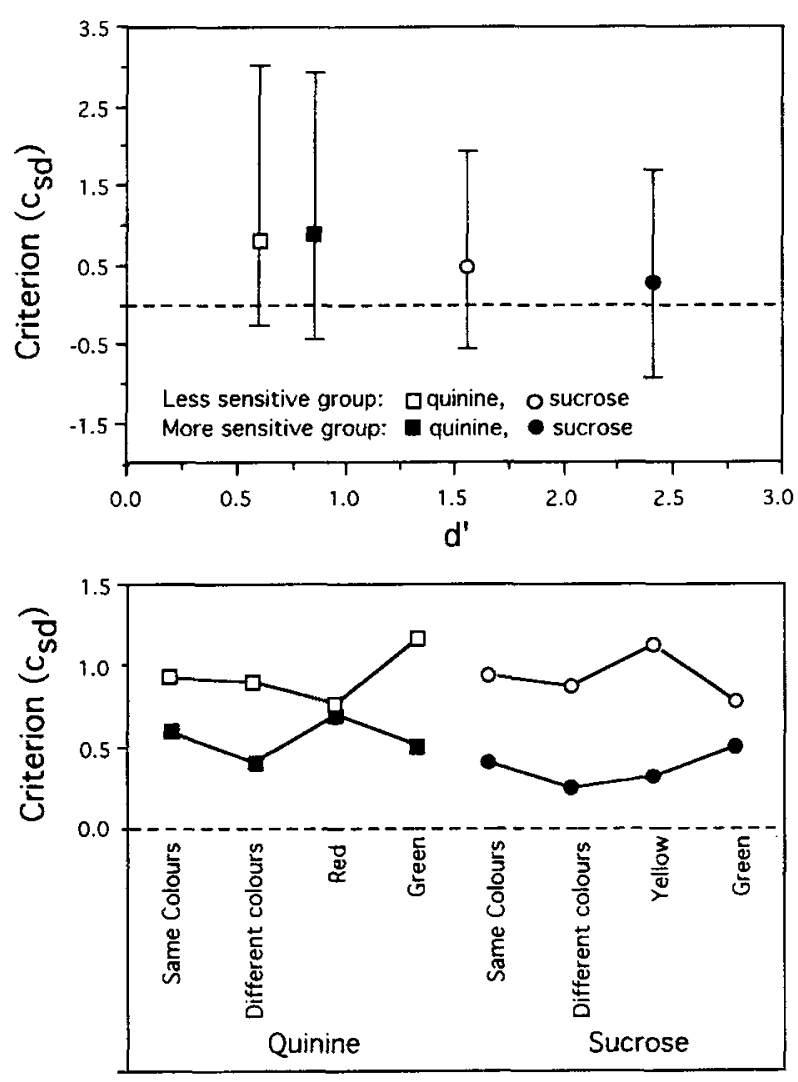

Figure 1. Criteria adopted by the less sensitive participants (open symbols) and the more sensitive participants (closed symbols). In the upper panel, criteria are shown as a function of $d^{\prime}$ for all comparisons involving quinine (squares) and sucrose (circles). The bars surrounding each datum represent the category range. In the lower panel, criteria are shown for various subsets of the data. The points are joined for visual purposes only.

meaning in both panels. Open symbols represent the 3 less sensitive participants, and closed symbols represent the 3 more sensitive participants. In the upper panel of the figure, the criteria the participants adopted over all comparisons using quinine (squares) and sucrose (circles) are shown versus the associated $d^{\prime}$ values. The bars around each datum represent the range between the most conservative and liberal response categories. In the bottom panel, the criteria of the participants are shown for various subsets of the data. The points are joined merely to facilitate visual comparison. The corresponding $d^{\prime}$ values can be ascertained from Table 1 . The biases exhibited by the less sensitive participants in the lower panel of Figure 1 are consistently more in favor of "same" responses than are those of the more sensitive participants $[t(14)=6.21$, $p<.05$, two tailed].

\section{Discussion}

Figure I clearly shows a preponderance of "same" responses. According to Macmillan and Creelman (1991), this is a common finding in same-different tasks. The top 
panel suggests a small decrease in the tendency toward "same" responses as sensitivity increases. The lower panel provides little support for an intuitive expectation that a greater range of variations in taste quality would be labeled "same" if pairs of solutions appeared visibly alike than if they appeared different. Neither dissimilarity in appearance nor particular colors were as effective in influencing responding as sensitivity was. For both taste qualities, less sensitive participants were more inclined toward "same" responses than were more sensitive participants, irrespective of whether pairs of solutions looked the same or different.

A stronger association between sensitivity and response bias than between visual appearance and response bias had not been anticipated. For this reason, the association between bias and sensitivity was explored using a different modality-hearing - in Experiment 2. The use of auditory stimuli allows sufficient trials to be undertaken to examine the behavior of individual listeners, over an extended range of task difficulties.

\section{EXPERIMENT 2}

In Experiment 2, participants were required to discriminate between pairs of $1000-\mathrm{Hz}$ sinusoids differing in amplitude. By contrast with Experiment 1, no other factors thought to influence responding were deliberately introduced. To facilitate comparison with the outcome of Experiment 1, the same-different rating task was used again, but sensitivity and bias were also measured using the more common single-interval yes-no rating task.

\section{Method}

\section{Participants}

Three graduate students at the University of Auckland, all men with normal hearing, participated. Their ages were 24, 29, and 32 years. Two were experienced in psychophysical auditory tasks. All completed at least 1,500 practice trials prior to data collection.

\section{Apparatus and Stimuli}

In both psychophysical tasks, a Hewlett-Packard 8904A multifunction synthesizer generated and delivered a continuous $1000-\mathrm{Hz}$ sinusoid to a gate. The sinusoid was shaped by a Hanning window and gated so as to produce $120-\mathrm{msec}$ signals inclusive of a $10-\mathrm{msec}$ rise and fall. A programmable attenuator subsequently determined the amplitude of each signal. The stimuli were presented to the left ear of each participant by way of TDH- 49 headphones fitted with MX-41/AR cushions. Calibration of the headphones was carried out using an artificial ear (Bruel and Kjaer, Model 4152) with a 6cc coupler. The participants were seated in a sound-attenuating booth (Amplaid model E), and responses were made on a computer keyboard.

The standard signal was a $1000-\mathrm{Hz}$ sinusoid at $57 \mathrm{~dB}$ SPL. Comparison signals were all higher in amplitude than the standard, and the differences ranged from $0.2 \mathrm{~dB}$ to $2.2 \mathrm{~dB}$ in the yes-no task and ranged from $0.6 \mathrm{~dB}$ to $2.6 \mathrm{~dB}$ in the same-different task. Within each range, stimulus amplitudes were separated by $0.4 \mathrm{~dB}$; thus, six amplitude differences were judged with each psychophysical paradigm. No background noise was added during stimulus presentations.

\section{Procedure}

Same-different Task. Experimental sessions consisted of blocks of 100 trials, plus 12 practice trials. Four blocks of 100 trials were completed for each of the six amplitude differences. At each amplitude difference, the four blocks of trials were completed within a single day. During each session, an outline of the task, including the rating scale, was displayed on a monitor to the participants. The sequence of events on a single trial was as follows: Every trial began with a 250 -msec warning LED followed after $500 \mathrm{msec}$ by two 120-msec observation intervals separated by $400 \mathrm{msec}$. The first and second observation intervals each contained a $1000-\mathrm{Hz}$ sinusoidal signal. If the standard amplitude is designated $A$, and the amplitude plus an increment is designated $B$, the four possible temporal orders for the presentation of the stimuli are $A A, B B, B A$, and $A B$. Each order was used equally often within a block of trials. Following each trial, the participant rated his confidence that the signals were of the same amplitude or of different amplitudes by typing a number from 1 (certain different) to 6 (certain same) on a keyboard. Responses were followed after $200 \mathrm{msec}$ by a 100 -msec feedback LED, which was either on the left if the amplitudes were different or on the right if the amplitudes were the same. The trial sequence was repeated after a delay of $300 \mathrm{msec}$.

Yes-no task. The sequence of events on a single trial was as follows: Every trial began with a 250-msec warning LED followed after $500 \mathrm{msec}$ by a $120-\mathrm{msec}$ signal. The signal was a $1000-\mathrm{Hz}$ sinusoid either at the standard amplitude or at the standard amplitude plus an increment. Within a block of trials, the size of the increment was constant. The standard or the standard-plus-increment was presented in pseudo-random order, with a probability of .5. Following each trial, the participant rated his confidence that the signal was of the smaller or larger amplitude and received feedback in the same manner as for the same-different task. All other experimental contingencies were the same as for the same-different task.

In both psychophysical tasks, the participants were informed of the a priori probabilities of the stimulus events.

\section{Results}

\section{Sensitivity}

Thirty-six ROCs were obtained in Experiment 2, representing the behavior of each of the 3 participants, at each of six amplitude differences, in both the yes-no task and the same-different task. Estimates of the sensitivity parameter $d^{\prime}$ were obtained by means of a maximum likelihood algorithm, as in Experiment 1. The normal-normal equal-variance model was adopted for the yes-no data, and, on the basis of the analyses reported below, the differencing model was adopted for the same-different data.

Two same-different models-the differencing model and the independent-observation model (see Noreen, 1981) - were fitted to the same-different data. The data departed significantly $(p<.01)$ from the independentobservation model for 5 of the 18 ROCs and differed significantly from the differencing model for 3 of the 18 ROCs ( 2 at $p<.05$, and 1 at $p<.01$ ). A comparison of the summed $\chi^{2}$ values [differencing model, $\chi^{2}(72)=102.67, p<.05$; independent-observation model, $\chi^{2}(72)=152.59, p<$ $.01]$ favored the differencing model.

An alternative way of distinguishing between the two same-different models was offered by Hautus, Irwin, and Sutherland (1994). Using this approach, the asym- 
Table 2

Best-Fitting Values of $d^{\prime}$ From Two Paradigms Measuring Discrimination Between Pairs of 1000-Hz Sinusoids Differing in Amplitude Along With $\boldsymbol{\chi}^{\mathbf{2}}$ Goodness-of-Fit Statistics

\begin{tabular}{|c|c|c|c|c|c|}
\hline \multirow[b]{2}{*}{$\Delta \mathrm{dB}$} & \multirow[b]{2}{*}{ Participant } & \multicolumn{2}{|c|}{ Same-Different } & \multicolumn{2}{|c|}{ Yes-No } \\
\hline & & $d^{\prime}$ & $\chi^{2}$ & $d^{\prime}$ & $\chi^{2}$ \\
\hline \multirow[t]{3}{*}{0.2} & 1 & & & 0.26 & 2.22 \\
\hline & 2 & & & 0.17 & 7.04 \\
\hline & 3 & & & 0.35 & 7.04 \\
\hline \multirow[t]{3}{*}{0.6} & 1 & 0.83 & 4.41 & 0.56 & $10.39 *$ \\
\hline & 2 & 0.72 & 1.79 & 0.78 & 2.62 \\
\hline & 3 & 1.20 & 5.75 & 0.52 & 5.57 \\
\hline \multirow[t]{3}{*}{1.0} & 1 & 1.34 & 7.00 & 1.14 & 1.49 \\
\hline & 2 & 1.24 & $11.09^{*}$ & 1.09 & 1.92 \\
\hline & 3 & 1.37 & 7.66 & 0.82 & 3.26 \\
\hline \multirow[t]{3}{*}{1.4} & 1 & 1.56 & 0.99 & 1.86 & 1.20 \\
\hline & 2 & 1.64 & 5.36 & 1.01 & 7.92 \\
\hline & 3 & 1.51 & 1.34 & 0.99 & 3.78 \\
\hline \multirow[t]{3}{*}{1.8} & 1 & 2.53 & 4.92 & 2.11 & $11.26^{*}$ \\
\hline & 2 & 2.40 & $16.65^{* *}$ & 1.83 & 3.52 \\
\hline & 3 & 2.27 & 3.77 & 1.43 & 5.60 \\
\hline \multirow[t]{3}{*}{2.2} & 1 & 3.02 & 2.67 & 2.44 & 1.15 \\
\hline & 2 & 2.90 & 2.52 & 1.55 & 1.34 \\
\hline & 3 & 2.73 & $13.06^{*}$ & 2.19 & 5.03 \\
\hline \multirow[t]{3}{*}{2.6} & 1 & 3.95 & 2.28 & & \\
\hline & 2 & 3.65 & 2.76 & & \\
\hline & 3 & 3.77 & 5.70 & & \\
\hline
\end{tabular}

Note-For $\chi^{2}$ values, $d f=4 .{ }^{*} p<.05 . \quad{ }^{* *} p<.01$.

metry of the empirical ROCs is estimated by fitting the unequal-variance yes-no ROC to the data. When plotted on normal-normal coordinates, the slope of the ROC indicates the degree of asymmetry. Slopes above one are consistent with the asymmetric differencing model, whereas slopes equal to one are consistent with the symmetric independent-observation model. When the data from Experiment 2 were examined this way, only 1 of the 18 slopes was found to be below one $(0.90)$. The mean slope, $1.19(S D=0.046)$, differed significantly from one $[t(17)=4.14, p<.001]$. In the light of these outcomes, the differencing model was adopted. Support for the use of the differencing model in the present experiment is in keeping with the findings of Hautus et al. (1994), who used a similar minimum uncertainty design with 4 observers and three amplitude differences.

Table 2 records the sensitivity estimates for each participant, for the same-different and yes-no tasks, together with the $\chi^{2}$ goodness-of-fit statistics. Five of the total of $36 \chi^{2}$ values were associated with a low probability; however, models that deviate from data to a greater extent than the cases reported here may be acceptable (e.g., Frick, 1996; Press, Flannery, Teukolsky, \& Vetterling, 1986). Considering the data as a whole, the models are a satisfactory fit.

Over the range of amplitude differences reported in Table 2 that are common to the two procedures, average $d^{\prime}$ estimates for the same-different task were higher than $d^{\prime}$ estimates for the yes-no task ( $d^{\prime}$ same-different $=$ $1.82 ; d^{\prime}$ yes - no $=1.35$ )

\section{Response Bias}

Estimates of response bias were obtained from collapsed ratings, as in Experiment 1 . In the left-hand panels of Figures 2 and 3 , the solid circles and continuous lines represent these estimates versus $d^{\prime}$ for each participant ( $c$ or $c_{\text {sd }}$ as appropriate). The dashed horizontal lines represent the point of intersection between the "same" distribution and either of the two "different" distributions (Figure 2) or between the distributions associated with the smaller and larger amplitudes (Figure 3). The bias indices $c$ and $c_{\text {sd }}$ are sometimes divided by $d^{\prime}$ to produce normalized indices, $c^{\prime}$ or $c_{\mathrm{sd}}^{\prime}$, representing the criterion as a proportion of the sensitivity distance. The measure $c^{\prime}$ has been evaluated against other bias measures and has received little support (Macmillan \& Creelman, 1990; Snodgrass \& Corwin, 1988). However, for comparison, criteria are presented in this form in the right-hand panels of Figures 2 and 3.

Although the use of a rating scale increases the accuracy of $d^{\prime}$ estimates, it does not allow for a simple characterization of bias. As in Experiment 1 , in order to obtain more information on the relationship between sensitivity and bias, we examined the range of criteria adopted by the participants at each signal level. In Figures 2 and 3 , the open symbols and dashed lines represent the locations of each participant's most liberal and most conservative category boundaries.

\section{Discussion}

The positive values of $c_{\text {sd }}$ in Figure 2 confirm a clear and consistent bias in the same-different task toward "same" responses. In Figure 3, the single-point bias estimates from the yes-no task represent a criterion location close to the intersection of the underlying distributions. At this location, $c=0$. In both figures, the estimates from the collapsed ratings appear unaffected by changes in sensitivity. For 2 of the participants ( $\mathrm{S} 1$ and $\mathrm{S} 3$ ), the range over which the criteria are spread declines with sensitivity. In Figure 2, this reduction is attributable more to a movement in the criterion adopted for "certain same" responses than to a movement in the criterion adopted for "certain different" responses. The right-hand panels of Figures 2 and 3 indicate that, for all 3 participants, the criteria occupy a greater proportion of the sensitivity distance when sensitivity is low.

With regard to the generally lower $d^{\prime}$ values from the yes-no task than from the same-different task, Viemeister (1970) suggested that participants in the yes-no task may base their observations on the difference between the current experience and a noisy stored reference. If this is the case, yes-no $d^{\prime}$ estimates should be increased by a factor of $\sqrt{2}$. Applying this factor to the yes-no data in Table 2 would bring the average values from the two tasks into close agreement. However, Creelman and Macmillan (1979) reported differences in the average values of $d^{\prime}$ from a frequency discrimination task that are less than would be expected on this basis. Their $d^{\prime}$ es- 


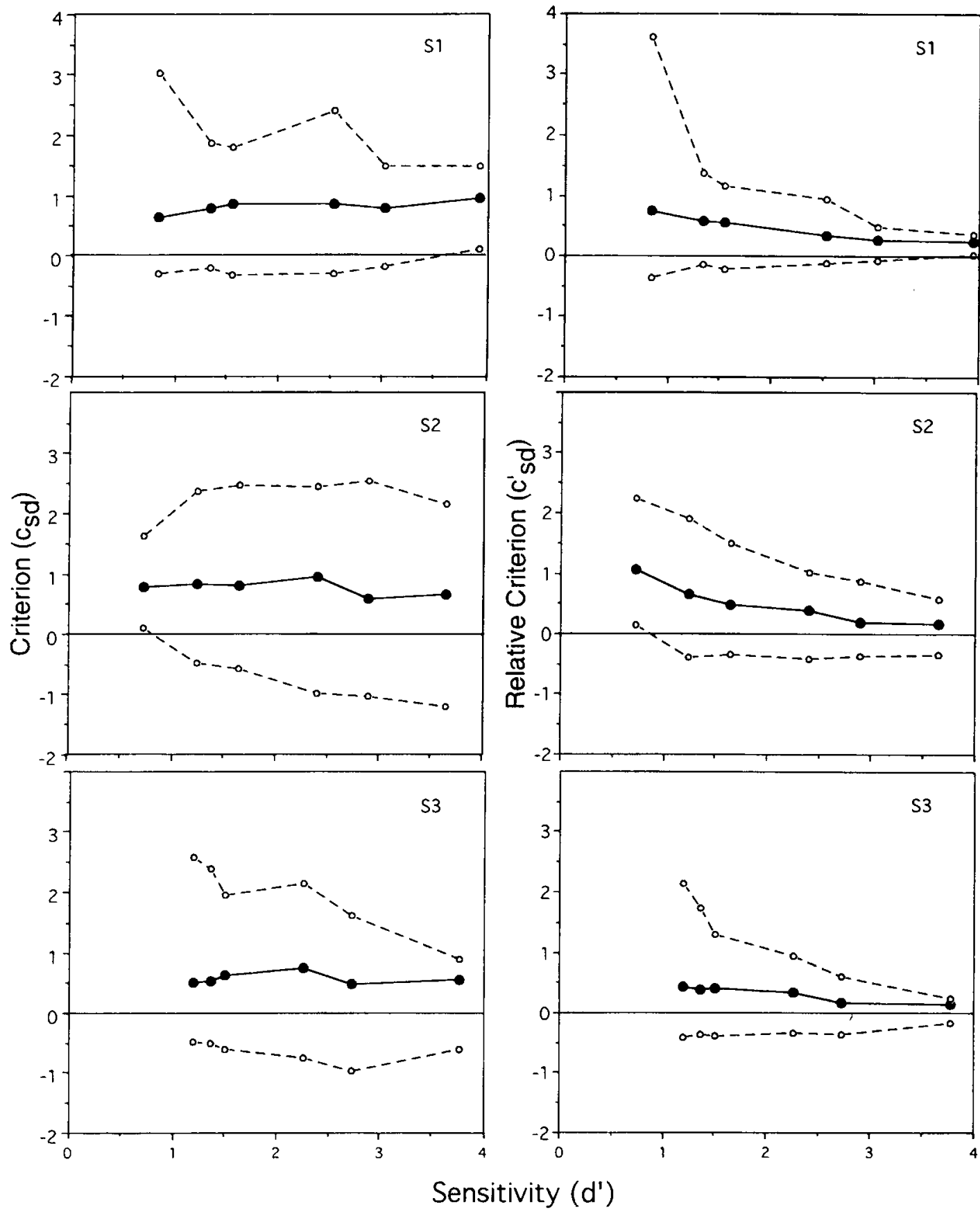

Figure 2. Each panel of the figure presents the data from 1 participant. The solid circles and continuous lines represent the bias estimates, $c_{s d}$, plotted versus $d$ '. The horizontal lines represent the point of intersection of the "same" and "different" distributions. Open circles and dashed lines represent the location of each participant's most liberal and most conservative category boundaries. The right-hand panels present the same data as in the left-hand panels, except that the bias index $\epsilon_{\mathrm{sd}}^{\prime}$ is used to represent the criterion as a proportion of the sensitivity distance, $d^{\prime}$.

timates were $18 \%$ higher in a same-different task than in a yes-no task. An additional contribution to such differences could be an inverse relationship between sensitivity and the length of the interobservation interval in twointerval paradigms (Durlach \& Braida, 1969; Kinchla \& Smyzer, 1967). Thus, it may be that correspondence between sensitivity estimates from yes-no and same- different tasks could be achieved by manipulating the length of this interval.

\section{GENERAL DISCUSSION}

In Experiment 1, we expected that a greater range of variations in sucrose or quinine would be labeled "same" 

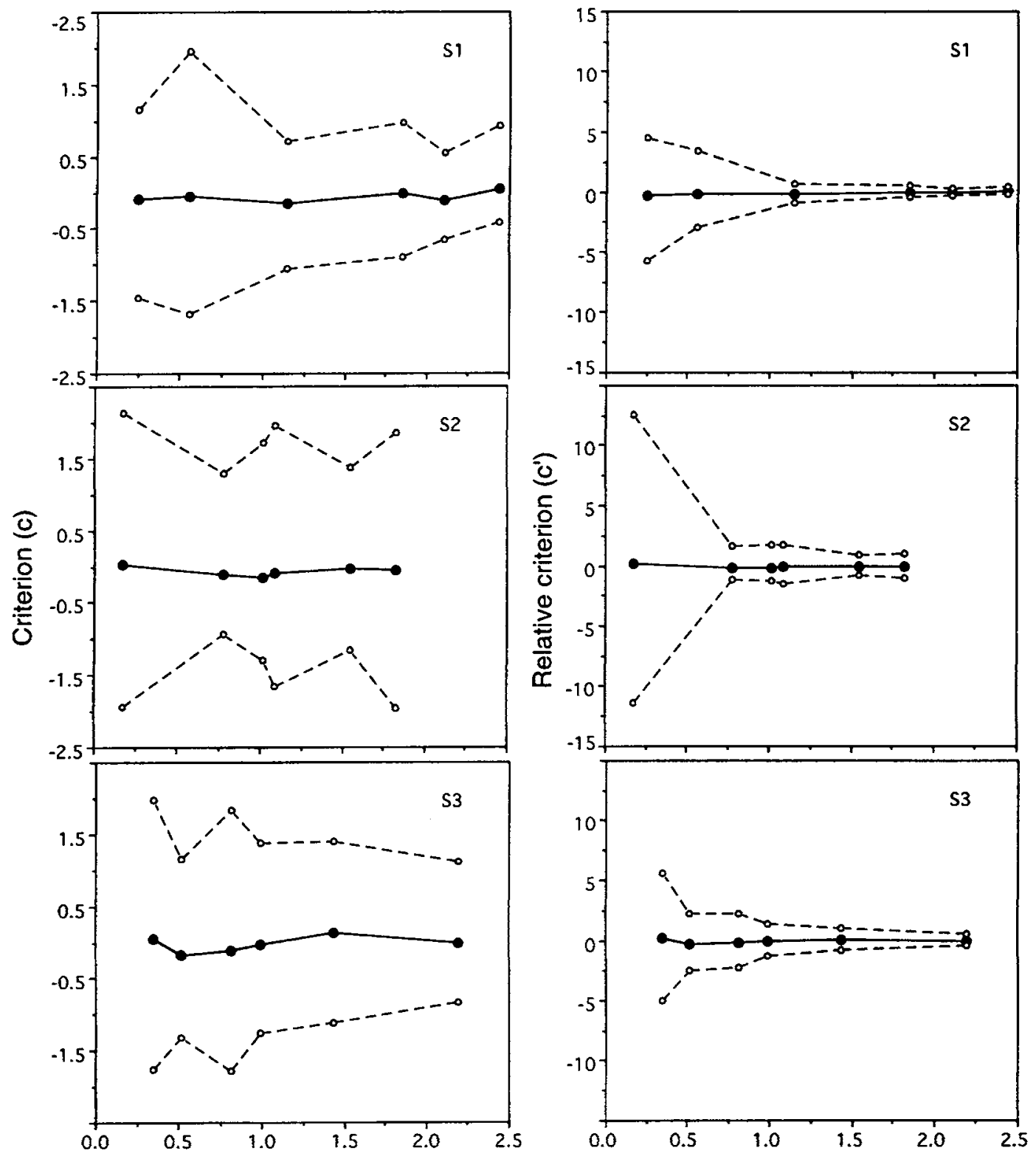

Sensitivity (d')

Figure 3. Each row of the figure presents the data from 1 participant in the yes-no auditory task. The left-hand panels show the bias estimates, $c$, plotted versus $d^{\prime}$. The symbols have the same meaning as in Figure 2 . The righthand panels present the same data, except that the bias index $c^{\prime}$ is used to represent the criterion as a proportion of the sensitivity distance, $d^{\prime}$.

if pairs of solutions appeared visibly alike than if they appeared different; however, there was scant evidence that this was the case. Instead, the less sensitive participants were more strongly inclined toward "same" responses than were the more sensitive participants, irrespective of whether pairs of solutions looked the same or different.

In Experiment 2, with auditory stimuli, there were no extrinsic reasons for biased responding. When the responses of individual participants were collapsed to ap- proximate the outcome of a categorical judgment, the estimates appeared unaffected by sensitivity. As in Experiment 1 , the participants in the same-different task were more inclined to respond "same" than to respond "different," irrespective of sensitivity.

A criterion is usually described as lying on a continuum from liberal (many hits and many false alarms) to conservative (few hits and few false alarms). However, this simple characterization of response bias does not apply to rating data, except when all the available infor- 
mation is assimilated into a single datum. To utilize more of the information inherent in the raw data, we examined the range over which the criteria were spread. The range used by the participants changed with sensitivity, albeit in somewhat idiosyncratic ways. Furthermore, particularly with respect to the yes-no data portrayed in Figure 3, the criteria representing the two most extreme response categories appear not to be independent. The lack of independence suggests a general alteration in response tendency such that the entire range over which the criteria are spread is either expanded or contracted. Thus, responses tend to become either more or less conservative at the lower end of the response range (ratings of 1 ) and, simultaneously, more or less liberal at the higher end of the response range (ratings of 6 ). In everyday terms, an increased range implies that participants require more evidence in favor of either stimulus class before using a confident rating. For this reason, we think it likely that, if simple binary decisions were called for, an increased range would translate into the adoption of a more conservative response bias, whereas a decreased range would translate into the adoption of a more liberal response bias. If this is so, it appears that the trial-by-trial history of subjective experiences with the stimuli is more influential than the known a priori probabilities of the alternative states of affairs. Such a phenomenon is in line with other empirical research suggesting a neglect of base rate information (Tversky \& Kahneman, 1982; however, see Koehler, 1996, for a discussion of the pros and cons of reliance on base rate information in realistic decision environments).

An examination of operant procedures with animals, in which reinforcement is rarely given for every response and alternative responses are often subject to different reinforcer rates, sheds only limited light on the performance of human observers in tasks such as those described here. Nevertheless, it is instructive to note that, in operant studies (e.g., Alsop \& Davison, 1991; McCarthy \& Davison, 1984), it has been demonstrated that when the physical disparity between stimuli is either very high or very low, the effects of stimulus discriminability and reinforcer ratio are not independent. That is, discriminability affects bias.

A reduction in the range of criteria with increasing sensitivity is not incompatible with the findings of See et al. (1997). In their Experiment I, a small correlation between $d^{\prime}$ and $c$ was uncovered, and the authors conceded that the theoretical independence they had assumed might not always hold empirically. In Experiment 2 of the same study, biases resulting from three different payoffs, when considered together, produced no main effects or interactions involving level of difficulty. However, the panels representing their preferred bias indices ( $c$ and $B_{\mathrm{D}}^{\prime \prime}$ ) in their Figure 3 suggest an influence of difficulty level that is particular to a neutral payoff. That is, in the absence of an externally imposed bias, more liberal re- sponding was evident with the easy discrimination than with the difficult discrimination.

Although general trends may exist, any association between sensitivity and response bias need not be universal. Indeed, the existence of idiosyncratic response biases is one reason why independent measures of sensitivity and bias are so desirable.

\section{CONCLUSION}

The existence of indices of discrimination that are independent of bias sometimes leads investigators to ignore the fact that measurement independence need not imply behavioral independence. Consequently, response biases are sometimes compared without regard to sensitivity. For this reason, it is important to consider what, if any, relationships exist between these quantities. Unfortunately, accurate and valid measures of each are difficult to obtain concurrently. On the one hand, single-point estimates of sensitivity, expressed as the detection theoretic index $d^{\prime}$, suffer a degree of imprecision relative to those obtained by fitting theoretical ROCs to a number of data points. On the other hand, response bias is often studied because of its relevance to decision making in everyday situations where simple categorical decisions, rather than confidence ratings, are the norm. An examination of the range of criteria used by the participants in the rating tasks undertaken in this study suggests that estimates of bias obtained from collapsed ratings might not adequately track changes in response bias. A general tendency toward more conservative response biases as sensitivity declines is supported by this and other studies and confirms that care is needed when making intergroup response bias comparisons. In order to establish the applicability of these findings to situations in which simple binary decisions are called for, it will be necessary to compare both rating and categorical judgments from the same individuals.

\section{REFERENCES}

Alsop, B.. \& DAvison, M. (1991). Stimulus disparity and the reinforcer ratio. Jotwnal of the Experimental Analysis of Behavior, 56, 67-80.

Crefiman, C. D.. \& Macmillan, N. A. (1979). Auditory phase and frequency discrimination: A comparison of nine procedures. Journal of Experimental Psichology: Human Perception \& Performance, 5 , $146-156$.

DONALDSON, W. (1992). Measuring recognition memory. Journal of Experimental Psychology: General, 121, 275-277.

DoRFMAN. D. D., \& ALF, E., JR. (1969). Maximum likelihood estimation of parameters of signal detection theory and determination of confidence intervals-Rating method data. Joumal of Mathematical Psvchologl, 6, 487-496.

DuBose, C. N., Cardell.o, A. V., \& Maller, O. (1980). Effects of colorants and flavorants on identification of perceived flavor intensity, and hedonic quality of fruit-flavored beverages and cake. Jomral of Food Science, 145, 1393-1415.

DuRlach, N. I., \& Braitia, L. D. (1969). Intensity perception: I. Preliminary theory of intensity resolution. Joumal of the Acoustical Society of America, 46, 372-383 
FRICK, R. W. (1996). The appropriate use of null hypothesis testing. $P_{s y-}$ chological Methods, 1, 379-390.

Hautus, M. J., \& IRwin, R. J. (1995). Two models for estimating the discriminability of foods and beverages. Journal of Sensory Studies, 10, 203-215.

Hautus, M. J., Irwin, R. J., \& Surherland, S. (1994). Relativity of judgements about sound amplitude and the asymmetry of the samedifferent ROC. Quarterly Journal of Experimental Psychology, 47A, 1035-1045.

Irwin, R. J., Hautus, M. J., \& Stillman, J. A. (1992). Use of the receiver operating characteristic in the study of taste perception. Journal of Sensory Studies, 7, 291-314.

Irwin, R. J., Stillman, J. A., Hautus, M. J., \& Huddeston, L. M. (1993). The measurement of taste discrimination with the samedifferent task: A detection-theory analysis. Journal of Sensory Studies, 8, 229-239.

ISHIHARA, S. (1964). Tests for color blindness. London: H. K. Lewis.

KinCHLA, R. A., \& SMYzER, F. (1967). A diffusion model of perceptual memory. Perception \& Psychophysics, 2, 219-229.

KOEHLER, J. J. (1996). The base rate fallacy reconsidered: Descriptive, normative, and methodological challenges. Behavioral \& Brain Sciences, 19, 1-53.

Macmillan, N. A., \& Creelman, C. D. (1990). Response bias: Characteristics of detection theory, threshold theory, and "nonparametric" indexes. Psychological Bulletin, 107, 401-413.

Macmillan, N. A., \& Creelman, C. D. (1991). Detection theory: A user's guide. Cambridge: Cambridge University Press.

Macmillan, N. A., \& Creelman, C. D. (1996). Triangles in ROC space: History and theory of "nonparametric" measures of sensitivity and response bias. Psychonomic Bulletin \& Review, 3, 164-170.

MaGA, J. A. (1974). Influence of color on taste thresholds. Chemical Senses \& Flavor, 1, 115-119.

MCCARThY, D., \& DAVISON, M. (1984). Isobias and alloiobias functions in animal psychophysics. Journal of Experimental Psychology: Animal Behavior Processes, 10, 390-409.

NOREEN, D. L. (1981). Optimal decision rules for some common psychophysical paradigms. In S. Grossbert (Ed.), Proceedings of the Symposium in Applied Mathematics of the American Mathematical Society and the Society for Industrial and Applied Mathematics (Vol. 13. pp. 237-279). Providence, RI: American Mathematical Society.

NORTON, W. E., \& JoHNSON, F. N. (1987). The influence of intensity of color on perceived flavor characteristics. Medical Science Research, 15, 329-330.
Pangborn, R. M., \& Hansen. B. (1963). The influence of color on discrimination of sweetness and sourness in pear-nectar. American Journal of Psychology, 76, 315-317.

Press, W. H., Flannery, B. P., Teukolsky, S. A., \& Vetterling, W. T. (1986). Numerical recipes: The art of scientific computing. Cambridge: Cambridge University Press.

Roth, H. A., Radle, L. J., Gifford, S. R., \& Clydesdale, F. M. (1988). Psychophysical relationships between perceived sweetness and color in lemon- and lime-flavored drinks. Journal of Food Science, 53, 1116-1119.

See. J. E., Warm, J. S., Dember. W. N., \& Howe, S. R. (1997). Vigilance and signal detection theory: An empirical evaluation of five measures of response bias. Human Factors, 39, 14-29.

Snodgrass, J. G., \& Corwin, J. (1988). Pragmatics of measuring recognition memory: Applications to dementia and amnesia. Journal of Experimental Psychology: General, 117, 34-50.

Stillman, J. A. (1993). Color influences flavor identification in fruitflavored beverages. Journal of Food Science, 58, 810-812.

Stillman, J. A., \& Irwin, R. J. (1995). Advantages of the samedifferent method over the triangular method for the measurement of taste discrimination. Journal of Sensory Studies, 10, 261-272.

Stilliman, J. A., \& Troscianko, T. (1996). Influence of color and sensitivity on response bias in the discrimination of sweet and hitter beverages. Unpublished manuscript.

Troscianko, T. (1979). Analysis of colour-scaling data. Journal of Color Research \& Application, 4, 225-228.

TrOSCIANKO, T. (1982). Saturation as a function of test-field size and surround luminance. Journal of Color Research \& Application, 7, 89-94.

TUORILA-OlLikainen. H. (1982). Pleasantness of colorless and colored soft drinks and consumer attitudes to artificial food colors. Appetite Intake, 3, 369-376.

TVERSKY, A., \& KAHNEMAN, D. (1982). Evidential impact of base rates. In D. Kahneman, P. Slovic, \& A. Tversky (Eds.), Judgment under uncertainty: Heuristics and biases (pp. 153-160). Cambridge: Cambridge University Press.

VIEMEISTER, N. F. (1970). Intensity discrimination: Performance in three paradigms. Perception \& Psychophwsics. 8, 417-419.

WALTERS, D. E. (1996). How are bitter and sweet tastes related? Trends in Food Science \& Technology, 7, 399-403.

(Manuscripi received December 31, 1998: revision accepted for publication January 26,2000 .) 